\title{
Development of a twin-spool turbofan engine simulation using the Toolbox for Modeling and Analysis of Thermodynamic Systems (T-MATS)
}

\author{
Alicia M. Zinnecker * \\ NERR Engineering, Parma Hts, OH, 44130, USA \\ Jeffryes W. Chapman ${ }^{\dagger}$ \\ Vantage Partners, LLC, Brookpark, OH, 44142 \\ Thomas M. Lavelle $\ddagger$ \\ and \\ Jonathan S. Litt $\S$ \\ NASA Glenn Research Center, Cleveland, OH 44135, USA
}

The Toolbox for Modeling and Analysis of Thermodynamic Systems (T-MATS) is a tool that has been developed to allow a user to build custom models of systems governed by thermodynamic principles using a template to model each basic process. Validation of this tool in an engine model application was performed through reconstruction of the Commercial Modular Aero-Propulsion System Simulation (C-MAPSS) (v2) using the building blocks from the T-MATS (v1) library. In order to match the two engine models, it was necessary to address differences in several assumptions made in the two modeling approaches. After these modifications were made, validation of the engine model continued by integrating both a steady-state and dynamic iterative solver with the engine plant and comparing results from steady-state and transient simulation of the T-MATS and C-MAPSS models. The results show that the T-MATS engine model was accurate within $3 \%$ of the C-MAPSS model, with inaccuracy attributed to the increased dimension of the iterative solver solution space required by the engine model constructed using the T-MATS library. This demonstrates that, given an understanding of the modeling assumptions made in T-MATS and a baseline model, the T-MATS tool provides a viable option for constructing a computational model of a twin-spool turbofan engine that may be used in simulation studies.

\section{Nomenclature}

Variables

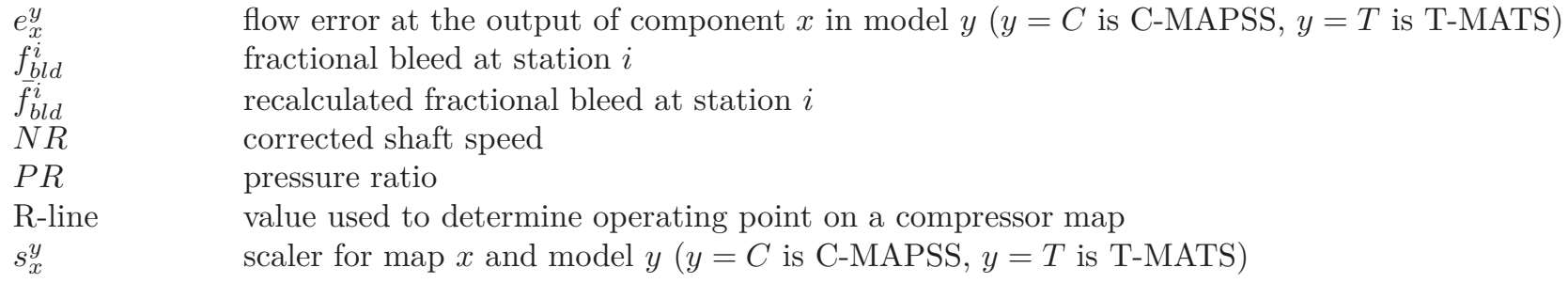

${ }^{*}$ Control Systems Engineer, alicia.m.zinnecker@nasa.gov, AIAA Member.

${ }^{\dagger}$ Controls Engineer, jeffryes.w.chapman@nasa.gov, AIAA Member.

$¥$ Aerospace Engineer, thomas.m.lavelle@nasa.gov.

$\S$ Research Engineer, Controls and Dynamics Branch, jonathan.s.litt@nasa.gov, AIAA Senior Member. 


$\begin{array}{ll}W_{f} & \text { fuel flow rate }(\mathrm{pps}) \\ W_{i} & \text { flow rate at station } i\end{array}$

$\begin{array}{ll}\text { comp } & \text { variable for compressor } \\ \text { cust } & \text { (fixed) customer bleed } \\ \text { des } & \text { fixed design value } \\ \text { map } & \text { variable interpolated from component map } \\ \text { sc } & \text { variable that has been scaled } \\ \text { turb } & \text { variable for turbine } \\ \text { un } & \text { variable that has been unscaled }\end{array}$

Acronyms

$\begin{array}{ll}\text { BPR } & \text { bypass ratio } \\ \text { C-MAPSS } & \text { Commercial Modular Aero-Propulsion System Simulation } \\ \text { C-MAPSS40k } & \text { Commercial Modular Aero-Propulsion System Simulation (40k) } \\ \text { HPC } & \text { high-pressure compressor } \\ \text { HPT } & \text { high-pressure turbine } \\ \text { IC } & \text { initial condition } \\ \text { IS } & \text { iterative solver (DIS = dynamic IS, SSIS = steady-state IS) } \\ \text { LPC } & \text { low-pressure compressor } \\ \text { LPT } & \text { low-pressure turbine } \\ \text { pps } & \text { pounds-mass per second } \\ \text { rpm } & \text { revolutions per minute } \\ \text { T-MATS } & \text { Toolbox for Modeling and Analysis of Thermodynamic Systems } \\ \text { VBV } & \text { variable bleed valve } \\ \text { VSV } & \text { variable stator vanes }\end{array}$

\section{Introduction}

$\mathrm{T}^{\mathrm{H}}$ HERE are numerous gas turbine engine simulations available, each with its own features that make it appropriate for specific applications. Those applications for which high frequency/high fidelity information is important may require a simulation with volume dynamics, while for lower frequency applications, simulations that only include speed and temperature dynamics, with the appropriate component performance characteristics, may suffice. ${ }^{1}$ The latter category is generally appropriate for control law design and gas path diagnostic algorithm development. ${ }^{2}$ The Modular Aero-Propulsion System Simulation (MAPSS), ${ }^{3}$ the Commercial Modular Aero-Propulsion System Simulation (C-MAPSS), ${ }^{4-6}$ and the Commercial Modular Aero-Propulsion System Simulation 40k (C-MAPSS40k), ${ }^{7,8}$ fall into the latter category. Features such as how components are modeled (lumped or stage-by-stage) or whether flows are modeled as zero- or higherdimensional also play a role in the fidelity of the simulation. ${ }^{9}$

Having a simulation readily available that captures the appropriate level of detail for a given task can be of great benefit, but if a suitable simulation cannot be found, or if the desired model does not exist, one may need to be created. Tools available to create customized engine models, component by component, include the Numerical Propulsion System Simulation (NPSS), ${ }^{9}$ and the new Toolbox for Modeling and Analysis of Thermodynamic Systems (T-MATS). ${ }^{10}$ Although similar in modeling approach (both tools utilize a modular, component-wise framework) and flexibility, NPSS and T-MATS offer different user experiences. NPSS offers a platform for modeling engines through a code-based interface, while T-MATS provides a more visual interface for model development. Additionally, T-MATS was developed as an open-source extension for use in the MATLAB/Simulink ${ }^{\circledR}$ (The MathWorks, Inc.) environment, completely removing the need for integration with this commonly-used simulation and modeling software, a time-consuming step of model development with NPSS. Unlike NPSS, however, T-MATS requires the user to provide engine data (e.g. maps and scaler) from other sources in order to run simulations. Despite the effort required to develop a new simulation, these tools offer a user the advantage of being able to define the level of fidelity in each component 
(for example, developing a multistage compressor model as opposed to a lumped parameter model).

When developing a simulation using such a tool, it is important to confirm that the model is working correctly, both to validate that the components were connected properly, and to validate the tool itself. This paper describes the process of validating a model constructed using T-MATS that duplicates the performance of the open-loop C-MAPSS (v2) engine model.

The engine plants used in this study are presented in Section II, with a discussion highlighting how the T-MATS model was modified to be able to replicate the C-MAPSS engine. Section III describes the integration of these plants with an iterative solver and validation of the steady-state and dynamic T-MATS models against the C-MAPSS model. The process for constructing these models using T-MATS, and the validation results, are summarized in Section IV.

\section{Description of engine models}

A generic twin-spool turbofan engine may be represented by the diagram in Fig. 1; this engine contains three compressors, two turbines, a burner, and two nozzles connected through a series of ducts. The T-MATS library contains blocks for modeling each component of an engine by providing information, such as maps and scalers, that specifies how that component operates. In this paper, the C-MAPSS (v2) engine model, described in Ref. 6, serves as a baseline for construction of a replica model using T-MATS. In the following discussion of the C-MAPSS and T-MATS engine plants, the assumptions about the maps, scalers, and flow errors in each model will be mentioned. It was important to address any differences in these assumptions through modification of the baseline T-MATS library blocks to ensure this model adequately replicated the C-MAPSS model. Step-by-step details of building the T-MATS engine plant model have been omitted as they exceed the scope of the paper; they may be found in the T-MATS User's Guide. ${ }^{10}$

\section{A. C-MAPSS engine plant model}

The C-MAPSS engine model is a computational model of a twin-spool turbofan engine that may operate in open- or closed-loop. It is implemented in Simulink, the graphical development and simulation environment of the widely-available MATLAB software tool, making it useful as a research platform for the controls and health-management community. ${ }^{4-6}$ The model captures representative dynamics of a twin-spool 90, $000 \mathrm{lbf}$ thrust-class turbofan engine which, as depicted in Fig. 1, contains a fan, low-pressure compressor (LPC), and low-pressure turbine (LPT) on one shaft, and a high-pressure compressor (HPC) and high-pressure turbine (HPT) on the other. In addition to these five components, the engine contains a burner, two nozzles, five ducts, and variable geometry. Locations along the engine flow path are indicated by the station numbers in the figure; these will be used throughout the discussion of the models and simulation results.

Although it may be operated in open-loop by providing a fuel flow input profile, the C-MAPSS engine is intended to operate in closed-loop with a controller that produces a fuel flow command to maneuver the engine along a user-defined input profile without violating physical or safety limits in the engine. This

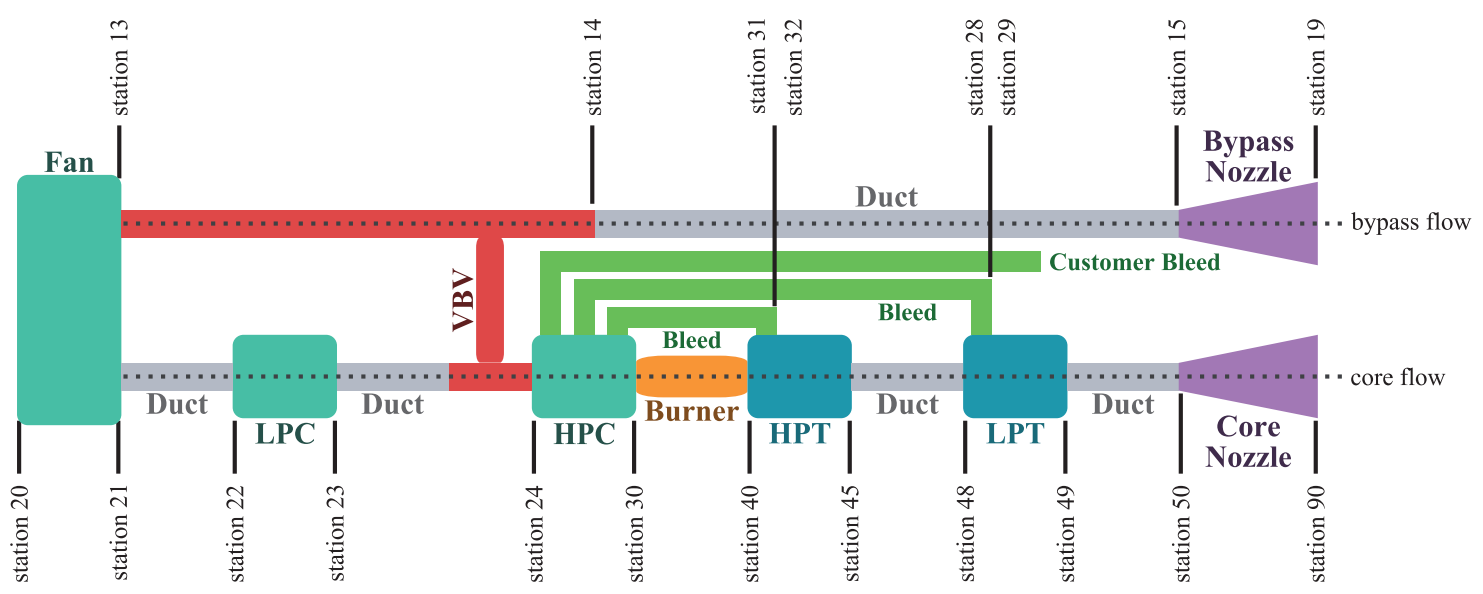

Figure 1. Block diagram of a twin-spool turbofan engine with stations numbered as assigned in the T-MATS engine model. 
profile contains information on how the environmental conditions (altitude, Mach number, and ambient temperature) and throttle change during a flight. In addition to fuel flow, the engine plant also requires inputs related to the variable geometry to be provided: the position of the variable stator vanes (VSV) in the HPC (not shown in Fig. 1) and the position of the variable bleed valve (VBV) located between the LPC and HPC. These inputs are nominally scheduled based on the (corrected) speeds of the core shaft and fan shaft, respectively. Off-nominal operation may be specified as part of the input profile, but is not considered in this study for simplicity.

Operation of the C-MAPSS engine is most affected by the three compressors (fan, LPC, and HPC) and two turbines (HPT and LPT). Each of these components is characterized by a set of maps relating (corrected) shaft speed, (corrected) flow rate, efficiency, and pressure ratio (PR) of the component and corresponding scalers to 'size' the maps based on a fixed design point. In this model, it is assumed that the (corrected) flow rate and efficiency interpolated from the maps scale according to the relationship in Eq. (1), which holds for both compressor and turbine maps.

$$
(\cdot)^{u n}=s_{(\cdot)}^{C}(\cdot)^{\text {map }}
$$

(Corrected) shaft speed, one of the variables used for interpolating the maps, scales similarly (Eq. (2)); for the compressor map, the scaler $s_{N R}^{C}$ in Eq. (2) is replaced by $s_{N R}^{C} / N R^{\text {des }}$, effectively adjusting the scaler based on a (fixed) design point, $N R^{\text {des }}$.

$$
N R^{m a p}=s_{N R}^{C} N R^{u n}
$$

The more-complex relationships in Eq. (3) are used, respectively, for unscaling (in a compressor) or scaling (in a turbine) the PR across each component.

$$
\begin{aligned}
P R^{u n} & =s_{P R}^{C}\left(P R^{\text {map }}-1\right)+1 \\
P R^{\text {map }} & =s_{P R}^{C}\left(P R^{u n}-1\right)+1
\end{aligned}
$$

These relationships are reflected in how the component maps themselves are constructed; if a different scaling relationship were desired, the corresponding map would have to be unscaled using the old relationship and rescaled to the design point using the new relationship.* This process allows for operation of the component to be unchanged under the new set of scaler relationships and will be utilized in construction of the T-MATS engine plant.

The unique operating point of a compressor is defined by the (corrected) shaft speed and R-line, used to interpolate (corrected) flow rate, efficiency, and pressure ratio from the component maps; for a turbine, (corrected) shaft speed and PR define the operating point and are used for map interpolation. As the engine moves through a flight profile, each compressor R-line and turbine PR must change to ensure that the flow out of the component is the same as the flow in. An iterative solver (IS) is used by C-MAPSS to reduce these flow errors to within a specified tolerance by adjusting the three R-lines and two PRs. The flow errors used by the IS are calculated for the HPC, HPT, LPT, core nozzle, and the section of the flow path between the fan and VBV. The formulation used to calculate the flow errors for the turbines indicates that the flow maps for these components only contain information on the main flow entering the turbine (that is, at stations 40 and 48) and not any of the bleed flow injected at the inlets (stations 31 and 28). The implications of this assumption, along with the scaling relationships for the compressor and turbine maps, will be addressed in the discussion of matching the T-MATS plant model to this baseline model.

\section{B. T-MATS engine plant models}

The T-MATS library may be used to construct an engine plant model in a modular fashion by combining blocks representing each component of the engine. In addition to the main components (compressors, turbines, nozzles, and burner), T-MATS contains blocks for ducts, valves, and a mechanism for 'splitting' the flow into a core (main) path and a bypass path (the effect of this mechanism is seen downstream of the fan in Fig. 1). Each block can be configured for a particular application by providing the necessary information through the block mask; this information may include performance attributes, such as maps, scalers, pressure losses, or physical attributes such as cross-sectional area or other constants, depending on the component. In providing these characteristics, it is important to know the assumptions made in the

*The iDesign feature in T-MATS can be used to automatically generate scalers, given a design point, for a relationship specified directly in the component code. ${ }^{10}$ 
code about how the component operates using this information; this was brought to the forefront during construction of the T-MATS engine plant model when using the maps, scalers, and other engine data from the C-MAPSS model. Note that the model referred to as the 'T-MATS engine plant model' only contains blocks for the engine components illustrated in Fig. 1; it does not contain an iterative solver.

In the T-MATS (v1) code, several assumptions are made that differ significantly from those made in the C-MAPSS model; in particular, the scaling of the maps and the information contained in the maps were not congruent between the models. Additionally, small differences, such as how bleed flow rates were calculated, were enough to contribute noticeably to mismatch between the models. With a goal of obtaining an engine plant model that was able to calculate the flow characteristics (flow rate, enthalpy, temperature, pressure, and fuel-to-air ratio) at each station in the engine to within 1\% of the C-MAPSS model, changes were needed in the information provided for configuring each component, and to the T-MATS code itself, to account for these assumptions.

Before considering changes at the code-level, recalculation of several maps and scalers was needed to meet the assumed scaler relationships in T-MATS. Unlike the C-MAPSS model, where the relationship between scaled (map) and unscaled map variables depends on the particular variable and component, TMATS assumes that each of the four map variables is scaled as in Eq. (1) (with $s_{(\cdot)}^{C}$ replaced by $\left.s_{(\cdot)}^{T}\right)$ for variables read from maps (e.g., (corrected) flow rate), and by the relationship represented in Eq. (2) (again with $s_{(\cdot)}^{T}$ replacing $s_{(\cdot)}^{C}$ ) for variables used for interpolation (e.g., (corrected) shaft speed). While the scaler relations for (corrected) flow rate and efficiency in the C-MAPSS model already had this form, it was necessary to recalculate scalers for the (corrected) shaft speed in the compressor map and for the pressure ratios in both compressor and turbine maps. The scalers for the (corrected) shaft speed were recalculated by absorbing the design value, $N R^{\text {des }}$, into the scaler specified for the compressors in the T-MATS plant $\left(s_{N R}^{T}=s_{N R}^{C} / N R^{d e s}\right)$. Modification of the scalers for PR was more involved as it required unscaling the PR maps for the compressors and turbines and calculating new PR scalers for the unscaled maps using the design values for each component, assuming the relationships suggested by Eqs. (1) (for the compressors) and (2)(for the turbines).

Recalculation of the maps and scalers for the compressors and turbines addressed most of the mismatch between the two engine plants; the small discrepancies that remained were related to the bleed flow from the HPC to the inlet and exit of the HPT ( $W_{31}$ and $W_{32}$, respectively, in Fig. 1). Comparison of the code implementing the HPC in each model revealed that, while the bleed flows to the inlet and exit of the LPT ( $W_{28}$ and $W_{29}$, respectively) were calculated the same way in each model $\left(W_{28,29}=f_{b l d}^{28,29} W_{24}\right.$, where $f_{b l d}^{28,29}$ specifies the fraction of inflow to the HPC that bleeds to the LPT), $W_{31}$ and $W_{32}$ were not. In the T-MATS HPC model, the fractional bleed rates $f_{b l d}^{31,32}$ are assumed to define the bleed flow rates to the HPT as fractions of the inflow to the HPC, $W_{24}\left(\right.$ like $f_{\text {bld }}^{28,29}$ ). In C-MAPSS, however, these bleed flow rates are assumed to be defined as fractions of the flow in the HPC after both LPT bleeds, $W_{28,29}$, and a fixed customer bleed, $W_{\text {cust }}$, have been removed (the first line in Eq. (4)). To account for the differing definitions of $f_{b l d}^{31,32}$, the fractional bleed rates must be recalculated for use in the T-MATS model:

$$
\begin{aligned}
W_{31,32} & =f_{\text {bld }}^{31,32}\left(W_{24}-W_{28}-W_{29}-W_{\text {cust }}\right) \\
& =f_{\text {bld }}^{31,32} W_{24}\left(1-f_{\text {bld }}^{28}-f_{\text {bld }}^{29}-\frac{W_{\text {cust }}}{W_{24}}\right) \\
& =\bar{f}_{\text {bld }}^{31,32} W_{24}-f_{\text {bld }}^{31,32} W_{\text {cust }}
\end{aligned}
$$

When $W_{\text {cust }} \neq 0$, there is a $1-2 \%$ difference in bleed flow rates $W_{31}$ and $W_{32}$ between the two plant models, exceeding the stated goal of matching the models with less than $1 \%$ error in all flow characteristics at all stations in the flow path. A closer look at the simulation results revealed that this additional error can be attributed to the term $f_{b l d}^{31,32} W_{\text {cust }}$ in Eq. (4): because customer bleed is fixed, it can not be represented as a constant fraction of $W_{24}$ and incorporated into the recalculated $\bar{f}_{\text {bld }}^{31,32}$. Analysis proceeds here under the assumption that $W_{\text {cust }}=0$ to avoid this discrepancy; it would be possible to modify how bleeds are handled in the T-MATS engine plant to allow for nonzero $W_{\text {cust }}$ by diverting the bleed, $W_{31,32}$, from the flow downstream of the compressor module.

The assumptions made in the models regarding the turbine maps and flow error calculations did not have an effect on the flow characteristics in the T-MATS plant model, but are more pronounced when an iterative solver (IS) is integrated with the plant. Although not influential in matching the T-MATS plant model to the C-MAPSS model, the modifications made to address these assumptions are included in this section. 
The T-MATS code assumed the turbine maps for (corrected) flow included the main inflow ( $W_{40}$ or $W_{48}$, for the HPT and LPT respectively) and inlet bleed ( $W_{31}$ and $W_{28}$, respectively), while the maps for the C-MAPSS model captured the inflow only. This assumption is manifested in the flow error calculations made in each model: given a flow rate interpolated from a turbine map, for a specific $N R^{s c}$ and $P R^{s c}$, C-MAPSS calculates the flow error as in Eq. (5), while T-MATS uses the expression in Eq. (6).

$$
\begin{gathered}
e_{H P T, L P T}^{C}=W_{48,50}-W_{40,48}-W_{31,28}-W_{32,29} \\
=W_{40,48}^{m a p}-W_{40,48} \\
e_{H P T, L P T}^{T}=W_{40,48}^{1}+W_{32,29}-W_{40,48}^{\text {map }} \\
=W_{40,48}+W_{31,28}-W_{40,48}^{\text {map }}
\end{gathered}
$$

The second equality in these equations holds because $W_{48,50}=W_{40,48}^{m a p}+W_{31,28}+W_{32,29}$ in the C-MAPSS model and $W_{40,48}^{1}=W_{40,48}+W_{31,28}$ in the T-MATS model. The final expressions in Eqs. (5) and (6) show that, by adjusting PR based on the flow errors, the IS drives the turbine operating condition to the case where the (corrected) flow rate interpolated from the maps is $W_{40,48}$ in the C-MAPSS model and $W_{40,48}+W_{31,28}$ in the T-MATS model. This difference must be accounted for at the code-level, made possible since T-MATS has been designed to allow for low-level modifications to accommodate differing assumptions in the modeling process.

The only assumption made about the solver architecture in the C-MAPSS engine model that was not reproduced in the T-MATS model was related to the calculation of the flow errors in the most-upstream region of the engine. The T-MATS engine plant requires two more solutions to be provided from the IS than required by the C-MAPSS plant, necessitating that two additional flow errors be calculated and provided to the IS when integrating it with the T-MATS plant. The modeling discrepancy inherent in this increased solver dimension replaces a solver input that indicates flow is balanced for a set of steady-state conditions $\left(e_{\text {fan }+L P C+V B V}^{C}=W_{20}-\left(W_{14}+W_{24}\right)\right)$ with three solver inputs $\left(e_{\text {fan }}^{T}, e_{L P C}^{T}\right.$, and $\left.e_{\text {bypass }}^{T}\right)$, one of which does not indicate balanced flow for the same steady-state conditions $\left(e_{\text {bypass }}^{T}\right.$, which is between $1.26 \%$ and $3.46 \%)$. When an IS is integrated with the T-MATS plant, a steady-state condition slightly different from the C-MAPSS model results, as will be shown in Section III.

\section{Comparing engine plant models}

The engine plant model constructed using the T-MATS block library was compared to the C-MAPSS engine model in two stages to verify the modifications made in the T-MATS engine plant model. Comparison of the two plant models was done by looking at the errors in flow characteristics (flow rate, enthalpy, temperature, pressure, and fuel-air ratio) at each station in Fig. 1; the T-MATS plant was said to 'match' the C-MAPSS model when these errors were each below $1 \%$. The validation was done by providing constant inputs, corresponding to the steady-state flow conditions of the C-MAPSS model when simulated with the inputs $W_{f}=1.67 \mathrm{pps}, 3.33 \mathrm{pps}, 4.17 \mathrm{pps}$, and $6.95 \mathrm{pps}$, to the T-MATS engine plant. Before validation of the T-MATS plant as an integrated system, each engine component was validated individually at these four steady-state conditions to check that the maps, scalers, and other engine data provided to the model were correct. This step allowed for mismatched assumptions, such as those made on map scaling, to be identified more readily by isolating possible sources of error to a single component. After this verification, the flow path was established by connecting the components to form the T-MATS plant model, which closely resembled the diagram in Fig. 1.

The T-MATS engine plant, which is not integrated with an IS, was similarly validated against the CMAPSS engine at the four steady-state flow conditions. The model inputs that would be provided by the IS (R-lines for fan, LPC, and HPC; PRs for HPT and LPT; $W_{20}$; and the calculated BPR) were specified in the same way as fuel flow and VBV position: as constant model inputs. The flow characteristics at each station were collected and compared to those obtained from simulation of the C-MAPSS model to verify that the T-MATS plant was within the $1 \%$ goal. The relative error of the flow rate, temperature, and pressure along the core flow path for $W_{f}=4.17$ pps are shown in Fig. 2 . These are representative results of the four simulations in that all results were well within $1 \%$ of the C-MAPSS results, suggesting that the T-MATS plant accurately replicates the C-MAPSS plant. It should be noted, however, that the flow errors calculated for these simulations suggest that the flow is not balanced in the T-MATS plant model, even though it is in the C-MAPSS model, due to the different flow error calculations. The effect of these assumptions can be studied further by integrating an IS with the T-MATS plant and performing additional validation. 

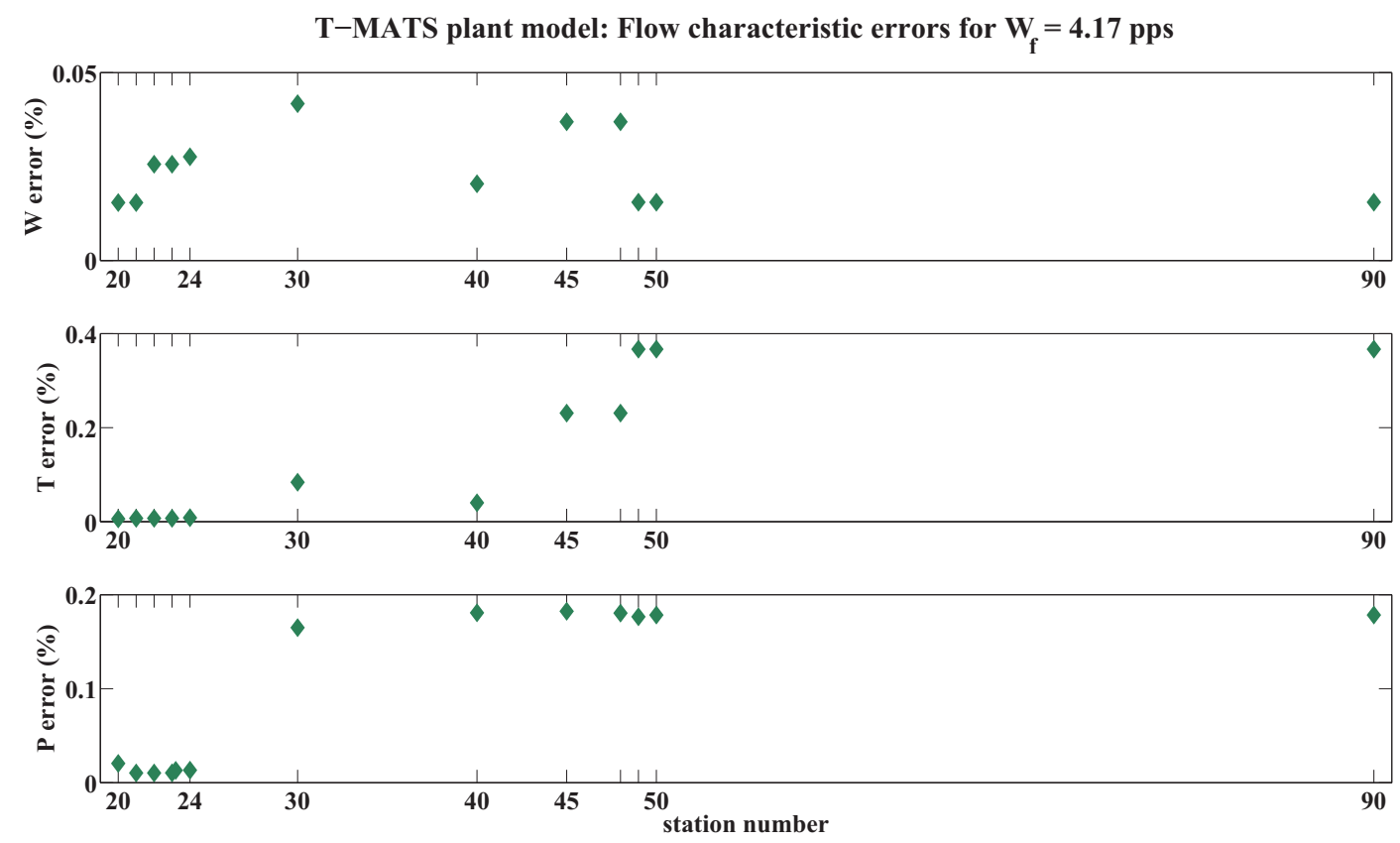

Figure 2. Percent errors in flow rate, temperature, and pressure at stations in the core flow path, calculated with respect to results from C-MAPSS, for simulation of the T-MATS engine plant with $W_{f}=4.17$ pps. All errors are less than 1\%, indicating the T-MATS plant accurately replicates the C-MAPSS plant.

\section{Evaluating the T-MATS models}

The T-MATS engine plant model discussed in Section II does not include a mechanism to ensure that mass and energy are conserved throughout a simulation, which is satisfied when the input and output flow of each component are within a specified tolerance of each other. An IS added to the model adjusts the operating conditions for the compressors (R-lines) and turbines (PRs) to 'balance the flow,' so the flow errors meet this tolerance. The T-MATS library contains two IS's that operate using the Newton-Raphson method: a steady-state IS (SSIS), which minimizes the flow errors for a constant input to the engine, and a dynamic IS (DIS), which drives the flow errors to zero at each time-step. (Mathematical detail of how these solvers work can be found in the T-MATS User's Guide. ${ }^{10}$ ) A version of the T-MATS model can be constructed by adding one of the two solvers to the plant model discussed in Section II. The required modifications to the structure of the Simulink model to accommodate these solvers are described in detail in the T-MATS User's Guide. ${ }^{10}$ Comparison of the simulation results from the T-MATS engine models with IS's to those from the C-MAPSS model can be used to demonstrate the utility of T-MATS in creating a Simulink-based model given component maps, scalers, and verification data.

Because both the C-MAPSS and T-MATS models are computational models, the flow characteristics at each station in Fig. 1 are known and can therefore be compared as part of the validation process. For this investigation, data from simulation of each model operating at sea-level static conditions $(0 \mathrm{ft}$ altitude, 0 Mach, standard-day temperature) for eight fuel flow profiles were compared. Four simulations, corresponding to the four constant fuel flow simulations listed in Table 1, were run to verify the T-MATS model when the plant was integrated with the SSIS (the 'steady-state T-MATS model'). The other four simulations, run with the single-step inputs in Table 1, tested the transient performance of the plant when integrated with the DIS (the 'dynamic T-MATS model'). The fuel flows in Table 1 were chosen to verify the T-MATS model over a large range of operating conditions (from 24,000 lbf to $88,000 \mathrm{lbf}$ of thrust production) in both steady-state and dynamic operation.

The IS in each model was configured as in Table 2. These settings specify limits on how much the Jacobian is perturbed, how much the solution can change at each time-step, the maximum number of iterations that can occur before the Jacobian is recalculated, and the conditions for which the solver will terminate its search for a solution. (The termination condition for the T-MATS model is smaller than for the C-MAPSS model because, unlike the C-MAPSS model (flow errors $O\left(10^{-12}\right) \%$ ), the solver in the T-MATS model produces 
Table 1. Fuel-flow inputs provided to the C-MAPSS engine model for generation of data used to evaluate the T-MATS engine models. Simulations included four constant fuel flows and four step-changes in fuel flow.

\begin{tabular}{|c|c|c|}
\hline Simulation & Initial fuel flow (pps) & Final fuel flow (pps) \\
\hline SS1 & 1.67 & 1.67 \\
\hline SS2 & 3.33 & 3.33 \\
\hline SS3 & 4.17 & 4.17 \\
\hline SS4 & 6.95 & 6.95 \\
\hline D12 & 1.67 & 3.33 \\
\hline D13 & 1.67 & 4.17 \\
\hline D24 & 3.33 & 6.95 \\
\hline D34 & 4.17 & 6.95 \\
\hline
\end{tabular}

Table 2. Iterative solver configuration for the C-MAPSS IS and for the T-MATS SSIS. Note that C-MAPSS uses a fixed Jacobian, while T-MATS calculates the Jacobian at the beginning of each set of iterations.

\begin{tabular}{|c|c|c|}
\hline Solver setting & C-MAPSS (D)IS & T-MATS SSIS \\
\hline Max Jacobian perturbation & N/A & 0.01 \\
\hline Max solution change per time-step (\%) & N/A & 2 \\
\hline Max iterations before Jacobian recalculation & N/A & 100 \\
\hline Max iterations for while-loop & 100 & N/A \\
\hline Termination condition (\%) & 2 & 1 \\
\hline
\end{tabular}

solutions with flow errors close to the termination condition.) In addition, initial conditions (IC's) need to be provided on a case-by-case basis due to the nonlinearity of the system. It should be noted that the IS in the C-MAPSS model is a dynamic IS that uses a fixed Jacobian, unlike the T-MATS IS's, which recalculate the Jacobian if convergence is not reached after a specified number of iterations; this may contribute to some of the mismatch observed in the simulation results presented here. In addition to presenting validation of the T-MATS model through the simulations listed in Table 1, the sensitivity of the solver to variations in configuration was also studied in an attempt to improve the accuracy of the T-MATS model; the results of this investigation will be presented briefly before the transient performance of the model with the DIS is discussed.

\section{A. Steady-state solver results}

The steady-state flow conditions resulting from simulation of the steady-state T-MATS model with the four constant fuel flow inputs listed in Table 1 were compared to the steady-state conditions of the CMAPSS model given those same inputs. The solutions of the SSIS and the steady-state shaft speeds and thrust production uniquely describe the operating conditions of each component in the engine plant model and, therefore, are representative of the flow characteristics in the model. A comparison of these results for the C-MAPSS and T-MATS models is shown in Table 3, where the error is calculated with respect to C-MAPSS. The most accurate results (those within $1 \%$ of C-MAPSS) were the shaft speeds, turbine PRs, and LPC R-line. Conversely, the steady-state fan R-line and BPR are the least-accurate solutions; these solutions in particular are most influenced by the difference in solution dimension between the C-MAPSS and T-MATS models.

The small differences between the turbine PRs and shaft speeds in the two models suggest that there should be relatively less error in flow characteristics for the HPT and LPT (stations 40 to 50). Specifically, the flow at these stations in the T-MATS model should be close to the flow in the C-MAPSS model since the maps are interpolated based on (corrected) shaft speed and PR. Likewise, the large error in fan R-line, along with the error in $W_{20}$, suggests that the flow rate in the T-MATS model in upstream stations is inaccurate with respect to the C-MAPSS model. The decrease in error in the LPC and HPC R-lines, combined with the large BPR error, indicates that the error in $W_{20}$ becomes concentrated in the bypass flow path downstream of where the flow splits. Even so, the inaccuracy in $W_{20}$ can be expected to affect all the stations in the 
Table 3. Percent error in steady-state solver results, shaft speeds, and thrust produced in the T-MATS model, calculated with respect to the C-MAPSS model. Note that $W_{20}$ and BPR are calculated from simulation results and are not part of the IS solution array in C-MAPSS.

\begin{tabular}{|c||c|c|c|c|}
\hline Variable & $W_{f}=1.67 \mathrm{pps}$ & $W_{f}=3.33 \mathrm{pps}$ & $W_{f}=4.17 \mathrm{pps}$ & $W_{f}=6.95 \mathrm{pps}$ \\
\hline Fan R-line & $4.2402 \%$ & $3.3082 \%$ & $3.1824 \%$ & $2.6227 \%$ \\
\hline LPC R-line & $0.4115 \%$ & $0.8505 \%$ & $0.8454 \%$ & $1.9311 \%$ \\
\hline HPC R-line & $1.3964 \%$ & $1.1804 \%$ & $1.5112 \%$ & $2.5628 \%$ \\
\hline HPT PR & $0.0382 \%$ & $0.0626 \%$ & $0.0592 \%$ & $0.0282 \%$ \\
\hline LPT PR & $0.7709 \%$ & $0.654 \%$ & $0.5419 \%$ & $0.7358 \%$ \\
\hline$W_{20}$ & $1.9178 \%$ & $1.2927 \%$ & $1.2474 \%$ & $0.5532 \%$ \\
\hline BPR & $3.1275 \%$ & $2.4064 \%$ & $2.2044 \%$ & $1.9136 \%$ \\
\hline$N_{f}(\mathrm{rpm})$ & $0.7043 \%$ & $0.311 \%$ & $0.2625 \%$ & $0.3203 \%$ \\
\hline$N_{c}(\mathrm{rpm})$ & $0.2403 \%$ & $0.2899 \%$ & $0.3193 \%$ & $0.369 \%$ \\
\hline$F_{g, \text { byp }}\left(\mathrm{lb}_{f}\right)$ & $2.6387 \%$ & $1.5826 \%$ & $1.3944 \%$ & $0.0534 \%$ \\
\hline$F_{g, \text { core }}\left(\mathrm{lb}_{f}\right)$ & $2.3795 \%$ & $1.7056 \%$ & $1.4905 \%$ & $0.8556 \%$ \\
\hline
\end{tabular}

engine, and is even reflected through the error in thrust, which is calculated based on the flow into each nozzle. These conditions are supported by the results in Fig. 3, which show the flow rate being the most inaccurate flow characteristic (up to $2 \%$ error with respect to the C-MAPSS model) at stations 20 to 21 (the fan), and maintaining an error around $1 \%$ at all other stations in the core flow, while the error in temperature and pressure remained below $1 \%$ at all stations (with exception at three stations when $W_{f}=6.95 \mathrm{pps}$ ). Not shown is the bypass flow path, which had errors in flow rates around $1.5 \%$ from the C-MAPSS results, much larger than the error in the core flow downstream of the fan.

Because the T-MATS plant model was verified using steady-state flow conditions from the C-MAPSS model, it was expected that integrating an IS with the T-MATS engine plant model would not cause the steady-state results to differ significantly from the C-MAPSS steady-state results. However, from Table 3 and Fig. 3, it can be seen that this was not the case; for a given (constant) input, the steady-state solution for the T-MATS model is close to, but not always within, $1 \%$ of the C-MAPSS solution. As discussed in Section II, the difference in calculation (and number) of the flow errors that comprise the input to the IS cause the T-MATS results to exceed the stated goal of $1 \%$ error. Before integration with an IS, the bypass nozzle flow error, which in the C-MAPSS model forms a single flow error with the fan and LPC, exceeds the error tolerance and therefore drives the IS to search the solution space for a steady-state condition where all seven flow errors are under 1\%. This is illustrated in Fig. 4, where the flow errors of the T-MATS engine before and after the SSIS has been integrated are shown with respect to the $1 \%$ error tolerance. These results suggest that the flow is not balanced in the T-MATS engine plant and show how the IS acts to do so by adjusting compressor R-lines, turbine PRs, $W_{20}$, and BPR to reduce the flow errors.

\section{B. Dynamic solver results}

The SSIS allows only for simulation of the system with constant inputs, as it iterates to converge the system to a steady-state operating condition by reducing the flow errors below the specified threshold. In order to perform simulations with inputs that change over time, the model must instead include the DIS, which iterates at each time-step to ensure the flow remains balanced throughout the simulation. Changes to the structure of the Simulink model necessary for integration of the DIS with the T-MATS plant model are not detailed here, but may be found in the T-MATS User's Guide. ${ }^{10}$ Two investigations were performed on the dynamic T-MATS model: studying the effects of changing the solver configuration settings from those listed in Table 2 and comparing the transient behavior of the C-MAPSS and T-MATS models using simulation with the step inputs in Table 1.

When the DIS is configured as specified in Table 2, results from simulation of the dynamic T-MATS model were nearly identical to those presented in Table 3 and Figs. 3 and 4, differing at most by $0.25 \%$ from the simulation results for the steady-state T-MATS model. It was of interest to study whether better matching to the C-MAPSS model could be achieved by changing the solver configuration from these baseline settings. A study of the eleven variations listed in Table 4 was performed, where a single change was made 

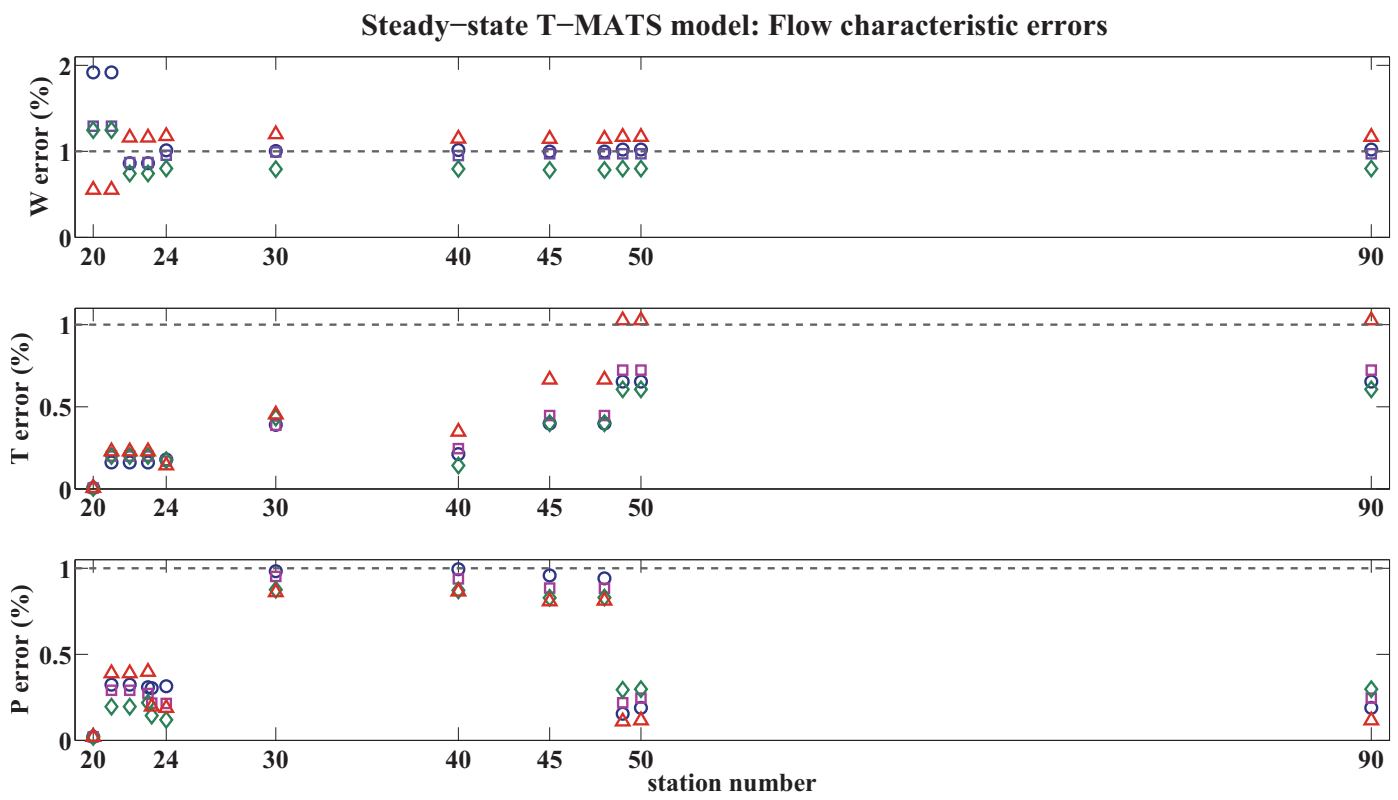

\begin{tabular}{|c|c|c|c|c|c|}
\hline$\circ W_{f}=1.67 \mathrm{pps}$ & & $\mathrm{W}_{\mathrm{f}}=3.33 \mathrm{pps}$ & $\diamond$ & $W_{f}=4.17 \mathrm{pps}$ & $\Delta W_{f}=6.95 \mathrm{pps}$ \\
\hline
\end{tabular}

Figure 3. Errors in flow rate, temperature, and pressure at each station in the engine, calculated with respect to C-MAPSS, for simulation of the steady-state T-MATS model with the constant inputs listed in Table 1. Comparison with Fig. 2 illustrates the effect of adding an IS to the model.

Flow errors for plant and steady-state T-MATS models
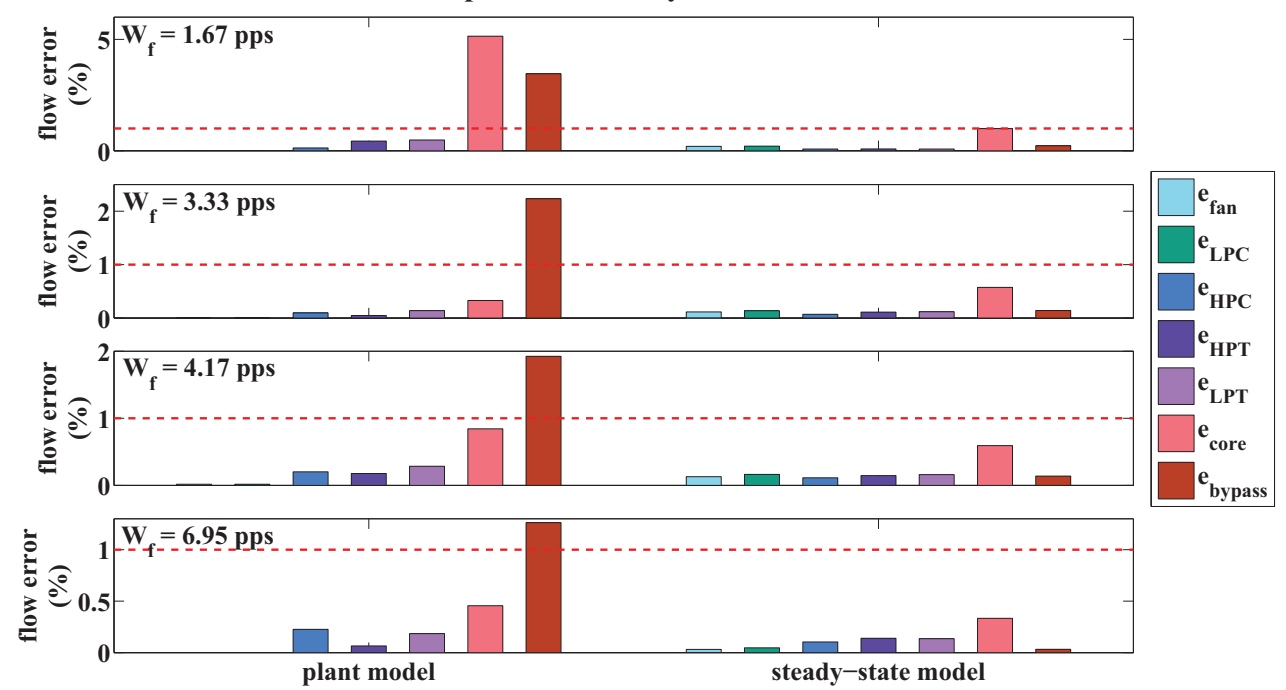

Figure 4. Comparison of the flow errors for each engine component in the T-MATS model without (left) and with (right) the SSIS integrated in the model. The error tolerance, here $1 \%$, is indicated by the dashed red line. 
Table 4. Iterative solver configurations studied for the dynamic T-MATS model simulation with the constantinputs listed in Table 1. The configuration chosen for simulation of the T-MATS model with step inputs is listed in the final column.

\begin{tabular}{|c|c|c|}
\hline Solver setting & Variations in T-MATS DIS & T-MATS DIS \\
\hline Max Jacobian perturbation & $0.001,0.05(0.0441$ for $6 \mathrm{k})$ & 0.04 \\
\hline Max solution change per time-step $(\%)$ & $0.5,5,10$ & 5 \\
\hline Max iterations before Jacobian recalculation & $15,500,1000$ & 100 \\
\hline Max iterations for while-loop & 100 & 100 \\
\hline Termination condition $(\%)$ & $0.1,2,5$ & 0.01 \\
\hline
\end{tabular}

from the baseline case for each simulation. The detailed implications of these choices are outside the scope of this paper; only general trends are presented here.

The trends observed through this investigation were used to guide a choice of solver configuration that resulted in a small improvement in how well the steady-state solution of the T-MATS model matched that of the C-MAPSS model. Decreasing the termination condition, increasing the maximum perturbation of the Jacobian, and increasing the maximum allowed change in the solution at each time-step generally resulted in a steady-state condition closer to that of the C-MAPSS model. (There was little effect on the solution by changing the maximum number of iterations between Jacobian recalculation.) Although this suggests that the impact of changing the configuration settings of the DIS on the accuracy of the dynamic T-MATS model was minimal, some improvement was achieved by following these trends and choosing the settings in the last column in Table 4, which were used to run the remaining simulations to be discussed.

After verifying the steady-state behavior of the dynamic T-MATS model against the C-MAPSS model, it was necessary to compare the transient behavior of the two models through simulation with the step inputs listed in Table 1. Because of the time-dependence of these results, the number of stations in the engine, and the number of flow characteristics for which data were collected, it is not practical to show all of the simulation data here; instead, results representative of the general observations made in the model are shown, taken from simulation of the step input from $W_{f}=3.33$ pps to $W_{f}=6.95 \mathrm{pps}$. As with the steady-state T-MATS model, looking at the IS solutions provides a way to qualitatively describe the behavior of flow characteristics during the simulations. Figure 5 shows the comparison of these solutions for the T-MATS and C-MAPSS models (solid and dashed lines, respectively). As observed for the steady-state T-MATS model (Table 3), the turbine PRs and shaft speeds match well between the two models, while the compressor R-lines, inlet flow $\left(W_{20}\right)$, and, especially, BPR in the T-MATS model were much less accurate. Since these solutions are near the corresponding steady-state solution in the time ranges where $W_{f}$ is constant (before the transition at 10 seconds and from a few seconds after the transition to the end of the simulation), this mismatch may be attributed to the differing approach to calculating flow errors. And like with the steady-state T-MATS model, these solver results suggest that the flow rate differs more than the other flow characteristics between the dynamic T-MATS model and the C-MAPSS model; this conclusion is supported by the results shown in Fig. 6 .

The results in Fig. 6 are similar to those in Fig. 3 in that they show pressure and temperature (generally) remaining within $1 \%$ of the C-MAPSS results throughout the simulation, while flow rates tend to exceed this value slightly, particularly following the transition in fuel-flow at 10 seconds. Flow characteristics at just four stations are shown: station 23, the exit of the LPC, station 30, the exit of the HPC, station 45, the exit of the HPT, and station 49, the exit of the LPT; many of the intermediate stations indicated in Fig. 1 are at duct exits, where the flow has only undergone a pressure reduction, so simulation data from these additional stations do not provide additional information. One of the most obvious features in the figure is the loss in accuracy of flow rate between stations 23 and 30 due to the presence of the VBV. This is most clear during the $3-4$ seconds following the transition in fuel flow. The likely reason for this mismatch is the way the VBV position is specified in each model: in the C-MAPSS model, a schedule is defined that maps the corrected core speed to the valve position, while in the T-MATS model the position is specified as an input profile, similar to fuel flow, using the steady-state values from simulation of C-MAPSS. Consequently, the valve position in the T-MATS model does not change in response to change in the corrected shaft speed; the mismatch is most noticeable during the input transition because the prescribed VBV position changes linearly but corrected core speed does not. Although affecting all flow characteristics downstream of stations 

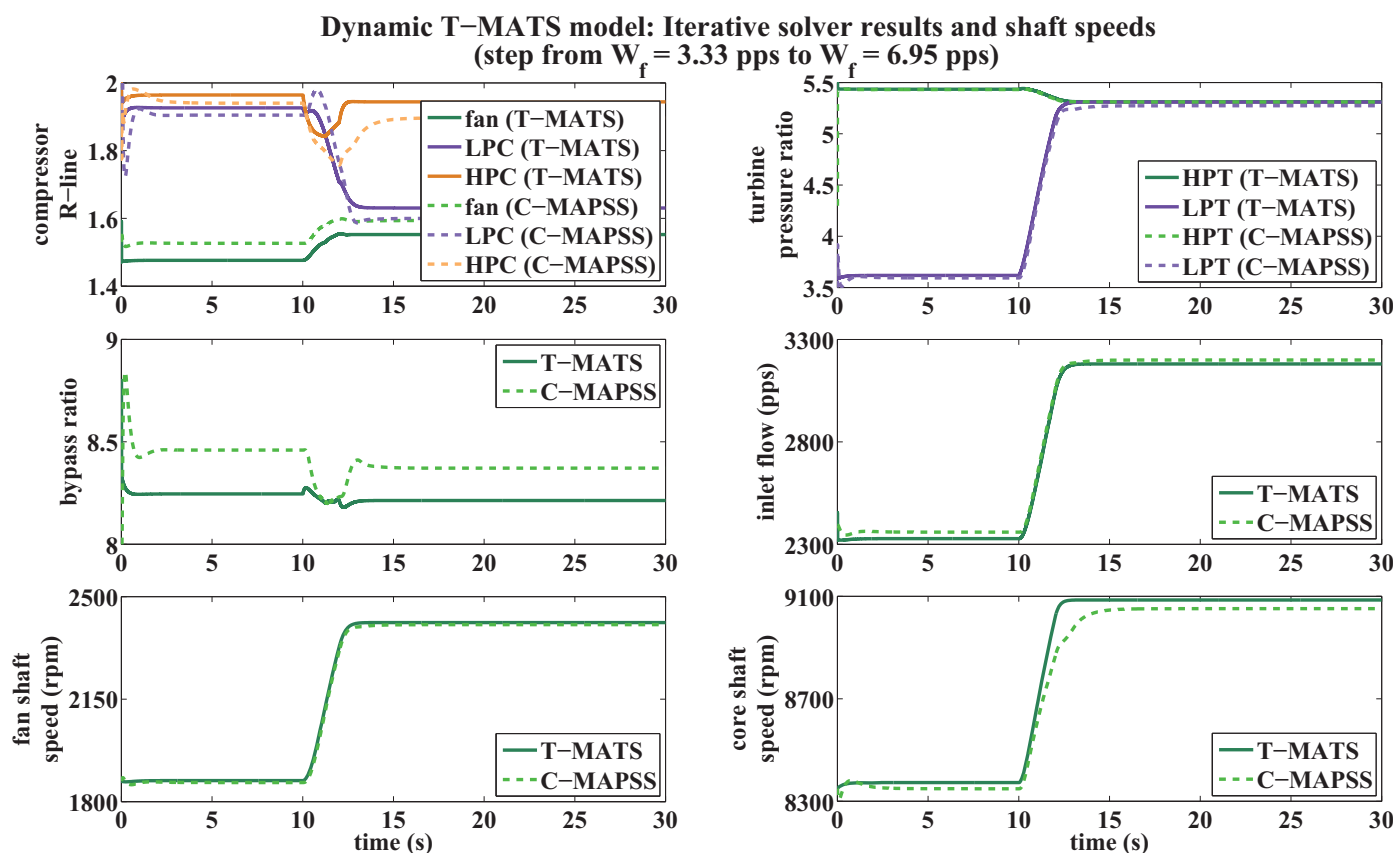

Figure 5. Comparison of DIS solutions, and shaft speed, for simulation of the dynamic T-MATS model and the C-MAPSS model with the step input from $W_{f}=3.33$ pps to $W_{f}=6.95$ pps.

24 and 14, this mismatch is not enough to produce significantly different conditions in the T-MATS model at the end of the simulation.

Additionally, it was observed that a large mismatch between the models occurred early in the simulation, attributed to the IC's specified for each model. The only IC's needed by the T-MATS model are for the IS and the integrators used for the shaft speeds, while the C-MAPSS model requires IC's to be specified for these and for the three inputs and six pressure and temperature sensors in the system. When possible, the initial conditions were specified identically for the two models, but the additional initial conditions required for the C-MAPSS model introduce the start-up transients seen in Fig. 6. After a few time-steps, the transient dies out as flow establishes a steady-state condition prior to the fuel flow transition.

Behind the results presented in Figs. 5 and 6 is the evolution of the flow errors as the DIS balances the flow at each time-step. From a high level, simulation of the models with a DIS progresses as follows: at the beginning of the time-step, the IS checks whether the flow errors are within the prescribed tolerance; if not, it iterates to find a solution that satisfies this condition before the simulation continues to the next time-step. For the dynamic T-MATS model, which has the solver configuration specified in Table 4, this is exemplified in Fig. 7, where the evolution of the seven flow errors is shown for the example simulation. The inset graphs in the figure, which show the iterations for $e_{H P C}^{T}, e_{L P T}^{T}$, and $e_{b y p}^{T}$ at each of 10 time-steps between 10.26 and 10.395 seconds, demonstrate the operation of the DIS. The dots represent solutions of the solver at each iteration while the solid lines illustrate how the flow error decreases on each iteration, until it is below the $0.01 \%$ tolerance (red dashed lines). The simulation then progresses to the next time-step (vertical dotted lines), where the error initially exceeds the tolerance and the solver must iterate again. The figure shows that, when the operating condition of the engine changes quickly (such as when provided a step change in $W_{f}$ ), several iterations are needed to balance the flow. Once the transient has died out (or $W_{f}$ changes less rapidly), the error is much closer to the tolerance at the beginning of the time-step and fewer iterations of the solver are required.

In addition to evaluating the internal flow characteristics of the T-MATS model against the C-MAPSS model, it is also of interest to compare the thrust production of each engine. As expected from the results in Figs. 5 and 6 , since thrust is a function of flow rate, the thrust produced by the two nozzles in the T-MATS model is within $1-2 \%$ of that calculated for the nozzle in the C-MAPSS model, as seen in Fig. 8.

Although the T-MATS model coupled with the IS did not meet the standard of having less than $1 \%$ error, the results in Figs. 3 to 8 and Table 3 demonstrate that both the steady-state and dynamic T-MATS 
Dynamic T-MATS Model: Flow characteristic errors in core path
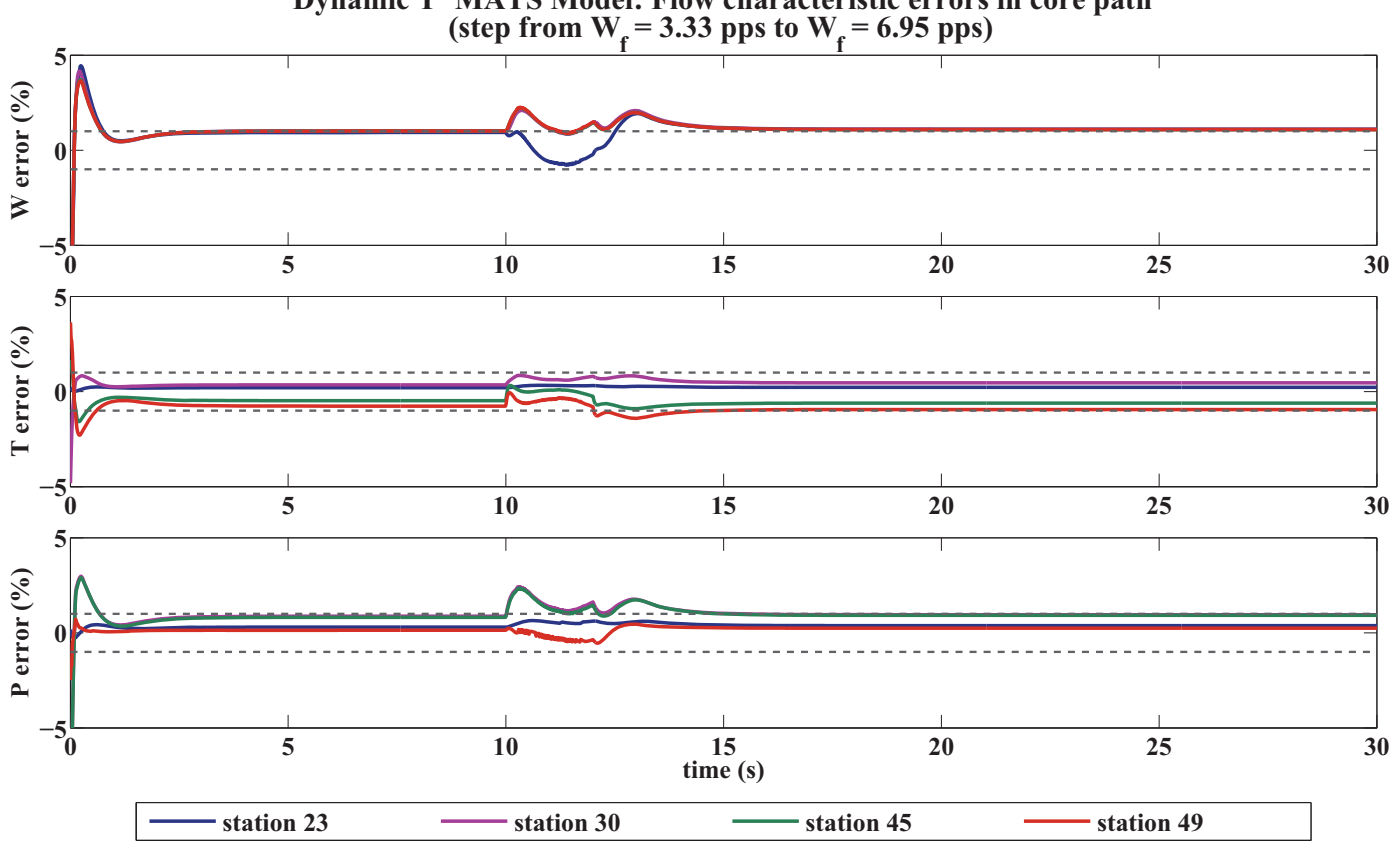

Figure 6. Errors in flow rate, temperature, and pressure at stations 23, 30, 45, and 49 (the exits of the LPC, HPC, HPT, and LPT, respectively) for the step input from $W_{f}=3.33$ pps to $W_{f}=6.95$ pps, calculated with respect to the C-MAPSS simulation results. The dashed lines represent an error of $1 \%$.
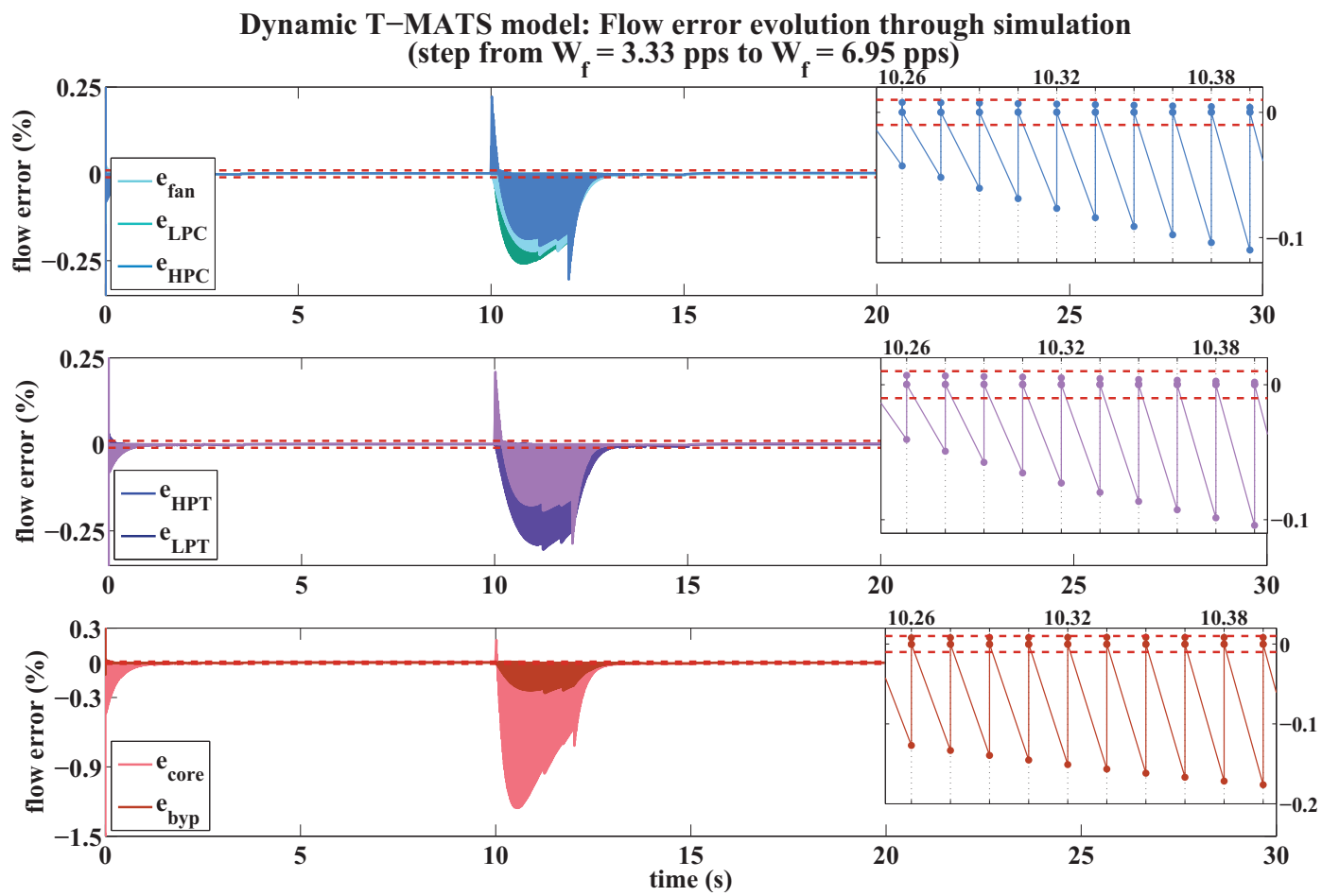

Figure 7. Evolution of the seven flow errors in the dynamic T-MATS model for simulation with the step input from $W_{f}=3.33 \mathrm{pps}$ to $W_{f}=6.95 \mathrm{pps}$. The edge of the color region shows the flow error at the beginning of each time step, which is reduced below the $0.01 \%$ tolerance (red dashed lines) by the DIS, as highlighted for $e_{H P C}^{T}, e_{L P T}^{T}$, and $e_{b y p}^{T}$ in the inset graphs. 

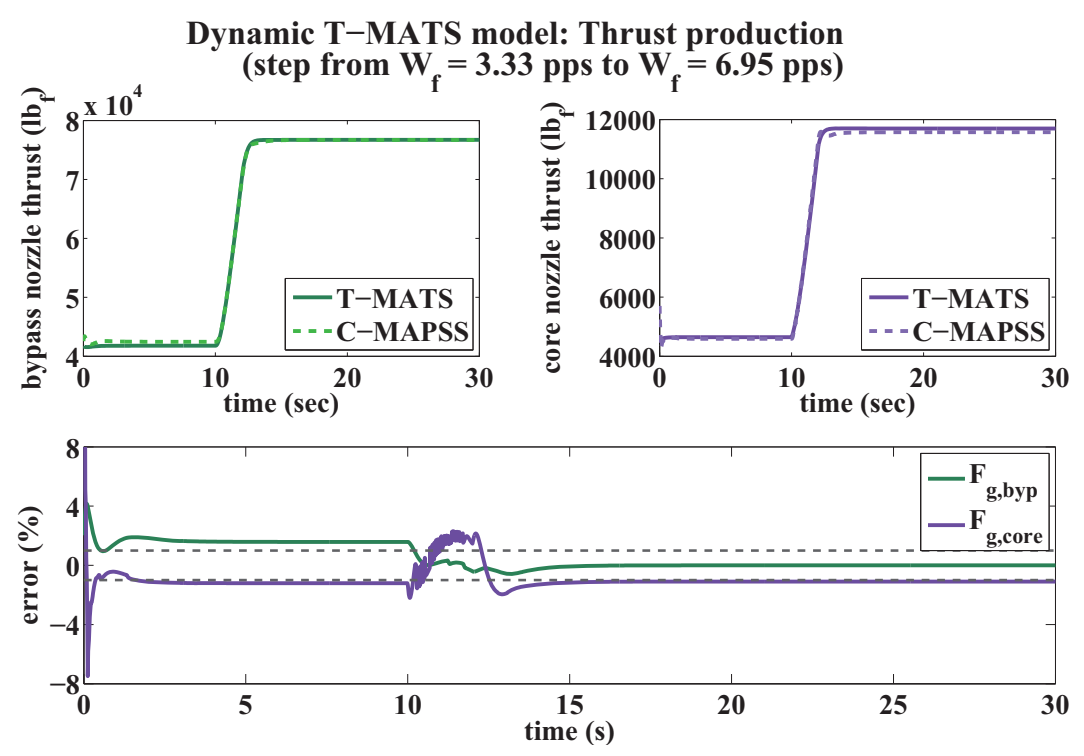

Figure 8. Comparison of the calculated thrust production of the bypass and core nozzles in the dynamic T-MATS model and the C-MAPSS model for simulation with the step input $W_{f}=3.33$ pps to $W_{f}=6.95$ pps.

models are still relatively accurate, within $2 \%$ of the C-MAPSS model. The main reason for this mismatch is due to the increased size of the solution space in the T-MATS model, required by the additional information provided by the solver to the T-MATS engine plant.

\section{Summary, conclusion, and future work}

An accurate replica of the C-MAPSS twin-spool turbofan engine model, limited to open-loop operation, has been developed using the Toolbox for Modeling and Analysis of Thermodynamic Systems (T-MATS). The process of constructing and validating this model emphasized the need to understand the original assumptions during construction of the baseline model. This knowledge led to and understanding of how the T-MATS model may need to be modified to meet a desired design approach or a specific design assumption, as the tool was developed to be modified on an application-specific basis. ${ }^{10}$ It was not only necessary to rescale some of the engine data provided to the T-MATS model, but also to make a code-level change to ensure the turbine flow error calculation accurately reflected the information contained in the component map.

Discussion of the T-MATS models focused on engine plant model and the integration of this model with an IS. However, it was useful to begin the modeling process by validating individual component models using the steady-state results from simulation of the baseline engine (C-MAPSS). Doing so allowed for isolation of specific components affected by modeling assumptions that differed between the models; it was also helpful to look at the component flow errors, both for these individual models and the plant model, in trying to identify the effect of some of these assumptions (e.g., before modifications were made to the turbine code in T-MATS, $e_{H P T}^{T}>10 \%$ and $e_{L P T}^{T}>4 \%$; these errors were reduced to $<0.5 \%$ after modifications were made). Once these assumptions were addressed in the T-MATS plant model, validation could continue through evaluation of this plant integrated with a steady-state or dynamic IS.

Simulation of both the steady-state and dynamic T-MATS models, with a set of constant and step fuel flow inputs, showed that the C-MAPSS model was accurately replicated, as most of the flow characteristics were within $1 \%$ of the C-MAPSS results for the same inputs; only the flow rate exceeded this limit, remaining within $2 \%$ of the C-MAPSS results. This suggests that the changes made to the engine data, by rescaling some maps and recalculating some scalers, and modification to the flow error calculation in the turbine properly addressed the different modeling assumptions made in the two models. Validation of the dynamic T-MATS model in open-loop may be expanded to include models with nonzero customer bleed and implementation of the VBV input thorough a scheduling scheme. Following this, the model should be validated in closed-loop with a controller similar to that used in the C-MAPSS simulation to further demonstrate the utility of the 
T-MATS tool.

\section{References}

${ }^{1}$ Kopasakis, G., Connolly, J. W., Paxson, D. E., and Ma, P., "Volume Dynamics Propulsion System Modeling for Supersonics Vehicle Research," Journal of Turbomachinery, Vol. 132, No. 4, October 2010, pp. 25-32.

${ }^{2}$ Simon, D. L., Propulsion Diagnostics Method Evaluation Strategy (ProDiMES) User's Guide, NASA/TM-2010-215840, January 2010.

${ }^{3}$ Parker, K. I. and Guo, T.-H., "Development of a Turbofan Engine Simulation in a Graphical Simulation Environment," NASA/TM-2003-212543, August 2003.

${ }^{4}$ DeCastro, J. A., Litt, J. S., and Frederick, D. K., "A Modular Aero-Propulsion System Simulation of a Large Commercial Aircraft Engine," Proceedings of the 44th AIAA/ASME/SAE/ASEE Joint Propulsion Conference ES Exhibit, AIAA 2008-4579, Hartford, CT, July 2008.

${ }^{5}$ Frederick, D. K., DeCastro, J. A., and Litt, J. S., User's Guide for the Commercial Modular Aero-Propulsion System Simulation (C-MAPSS), NASA/TM-2007-215026, October 2007.

${ }^{6}$ Liu, Y., Frederick, D. K., DeCastro, J. A., Litt, J. S., and Chan, W. W., User's Guide for the Commercial Modular Aero-Propulsion System Simulation (C-MAPSS), version 2, NASA/TM-2012-217432, March 2012.

${ }^{7}$ Csank, J., May, R. D., Litt, J. S., and Guo, T.-H., "Control Design for a Generic Commercial Aircraft Engine," Proceedings of the 46th AIAA/ASME/SAE/ASEE Joint Propulsion Conference, AIAA-2010-6629, Nashville, TN, July 2010.

${ }^{8}$ May, R. D., Csank, J., Lavelle, T. M., Litt, J. S., and Guo, T.-H., "A High-Fidelity Simulation of a Generic Commercial Aircraft Engine and Controller," Proceedings of the 46th AIAA/ASME/SAE/ASEE Joint Propulsion Conference, AIAA-20106630, Nashville, TN, July 2010.

${ }^{9}$ Lytle, J. K., "The Numerical Propulsion System Simulation: An Overview," NASA/TM-2000-209915, June 2000.

${ }^{10}$ Chapman, J. W., Lavelle, T. M., May, R. D., Litt, J. S., and Guo, T.-H., Toolbox for the Modeling and Analysis of Thermodynamic Systems (T-MATS) User's Guide, NASA/TM-2014-216638, January 2014. 